\title{
Avaliação da Resiliência como ferramenta para entender a fronteira amazônica como um sistema socioecológico
}

\author{
Resilience Assessment as a tool for \\ understanding the Amazon frontier as a \\ social-ecological system
}

\author{
Robert Buschbacher ${ }^{\mathrm{a}}$ \\ Simone Athayde ${ }^{b}$ \\ Wendy-Lin Bartelsc \\ Ricardo Mello ${ }^{d}$ \\ ${ }^{a}$ Amazon Conservation Leadership Initiative, \\ University of Florida, Gainesville, FL, EUA \\ End. Eletrônico: rbusch@ufl.edu \\ ${ }^{\mathrm{b}}$ Tropical Conservation and Development Program, \\ University of Florida, Gainesville, FL, EUA \\ End.Eletrônico:simonea@ufl.edu \\ 'School of Forest Resources and Conservation, Research Assistant Scientist, \\ University of Florida, Gainesville, FL, EUA \\ End. Eletrônico: wendylin@ufl.edu \\ ${ }^{d}$ Coordenador do Programa Amazônia do WWF-Brasil, \\ Brasília, DF, Brasil \\ End. Eletrônico: ricardomello@wwf.org.br
}

doi:10.18472/SustDeb.v7n2.2016.15134

\section{RESUMO}

Este artigo apresenta o pensamento de resiliência e uma metodologia para avaliação desta que consiste em definir o sistema (pergunta-chave e limites do sistema no tempo e no espaço); olhando para a história (linha do tempo, fatores desencadeantes e interações entre escalas); especificando a resiliência "do que" e "contra que" (definição de atributos e variáveis); cenários (prováveis fatores desencadeantes futuros e possíveis regimes futuros desejáveis e indesejáveis); e desenvolvimento de considerações de gestão para promover cenários desejáveis. A avaliação de resiliência de três grandes grupos sociais do município de Cotriguaçu em Mato Grosso, Brasil - proprietários de terras médias e grandes, os povos indígenas Rikbaktsa e agricultores familiares - revelou que cada grupo social passou por várias iterações do ciclo adaptativo (crescimento-colapso-reorganização), e que a dinâmica desse sistema é em grande parte determinada por fatores econômicos e políticos que vêm de uma escala 
maior. Contribuições para a metodologia de avaliação de resiliência incluem basear a análise do sistema em sua trajetória histórica, procurar incorporar explicitamente as perspectivas dos atores locais, e usar a análise de cenários para desenvolver possíveis intervenções de gestão.

Palavras-chave: Resiliência. Sistemas socioecológicos. Fronteira Amazônica. Avaliação da Resiliência. Fatores Desencadeantes. Atributos. Cenários.

\section{ABSTRACT}

This article introduces resilience thinking and a methodology for Resilience Assessment that consists of defining the system (key issue and system boundaries in time and space); looking at history (timeline and cross-scale drivers and interactions); specifying resilience of what to what (defining attributes and variables); scenarios (likely future drivers and possible desirable and undesirable future regimes); and developing management considerations to promote desirable scenarios. Resilience assessment for three major social groups of Cotriguaçu municipality in Mato Grosso, Brazil - medium to large landholders, the Rikbaktsa indigenous people, and family farmers - found that each social group has gone through multiple iterations of the growth-collapse-reorganization adaptive cycle and that the dynamics of this system are largely determined by economic and policy drivers that come from a larger scale. Contributions to the resilience assessment methodology are to base the analysis of the system on its historical trajectory, seek to explicitly incorporate the perspectives of local actors, and use scenario analysis to develop possible management interventions.

Keywords: Resilience. Social-ecological systems. Amazon frontier. Resilience Assessment. Drivers. Attributes. Scenarios.

\section{INTRODUÇÃO: PENSAMENTO DA RESILIÊNCIA E SUA RELEVÂNCIA PARA CONSERVAÇÃO E DESENVOLVIMENTO}

A Amazônia é uma região complexa, onde as atividades humanas, tanto sociais quanto econômicas, afetam e são afetadas por fatores biofísicos como a água, o clima e a biota. Considerando os desafios interrelacionados de tanto satisfazer as necessidades humanas quanto manter os serviços ecossistêmicos, e as interações entre as variáveis humanas e biofísicas em múltiplas escalas, é útil conceituar a Amazônia como um sistema socioecológico interligado (BUSCHBACHER, 2014; REDMAN et al., 2004; LIU et al., 2007). Grandes mudanças catalisadas pelo primeiro boom do extrativismo da borracha no final do século XIX, a construção da rodovia Belém-Brasília na década de 1960, o projeto Grande Carajás na década de 1980, e muitas outras forças, fazem da Amazônia um sistema socioecológico particularmente dinâmico.

Uma característica fundamental de todos os sistemas complexos é o seu potencial de mudança imprevista e rápida (não linear), o que frequentemente resulta de processos de feedback positivo (BUSCHBACHER, 2014). A Amazônia tem sido caracterizada como uma fronteira contestada onde grupos concorrentes reivindicam terra, florestas e recursos minerais, com estes frequentemente em condições de livre acesso e com governança limitada (SCHMINK; WOODS, 1992; LITTLE, 2001). Enquanto investimentos maciços em infraestrutura continuam na Amazônia (FEARNSIDE; LAURENCE, 2012), há indícios de um possível "ponto de inflexão" que poderia acabar com o processo de expansão contínua da fronteira em áreas de livre acesso (BROWDER et al., 2008; NEPSTAD et al., 2009; DAVIDSON, 2012). Por exemplo, as taxas de desmatamento diminuíram drasticamente desde 2004 (NEPSTAD et al., 2009), o sistema de Áreas Protegidas cobre agora 43,9\% da Amazônia Legal (VERÍsSIMO et al. , 2011) ${ }^{1}$, a posse da terra está sendo regularizada (BRITO; BARRETO, 2011), um menor número de assentamentos de reforma agrária estão sendo criados (OJEDA, 2012), a urbanização está aumentando (SIMMONS et al., 2002), e o agronegócio está evoluindo de um modelo de expansão espacial para outro de intensificação da produção (MACEDO et al., 2012). O "fechamento da fronteira" - como ocorreu na América do Norte no final do século XIX, quando a expansão para o oeste se consolidou, e que foi considerado um importante ponto de inflexão na história americana (TURNER, 1893) - poderia alterar drasticamente a dinâmica da Amazônia por meio da intensificação de conflitos fundiários sobre a terra restante e constranger as estratégias de uso da terra que dependem de colonização de terras de livre acesso. 
O argumento aqui não é que essas transformações sejam as mais prováveis, mas que existem vários futuros possíveis, imprevistos, que podem alterar radicalmente o contexto dos modos de vida sendo implementados por povos indígenas, colonos, madeireiros e fazendeiros ao longo das últimas décadas na Amazônia. Os atores desse sistema provavelmente serão desafiados pelas dificuldades de antecipar limiares, surgimento de surpresas, consequências inesperadas das mudanças climáticas, novas ondas de expansão de projetos de infraestrutura e a exacerbação de conflitos socioecológicos (LEVIN, 2005).

Os conceitos e pensamentos de resiliência podem contribuir para o manejo adaptativo dos sistemas socioecológicos (HOLLING, 1973; HOLLING; GUNDERSON, 2002; FOLKE, 2006). Aspectos-chave do pensamento de resiliência são reconhecer que a sociedade humana está incorporada em sistemas socioecológicos com fortes conexões entre processos antropogênicos (por exemplo, sociais, econômicos e políticos) e processos biofísicos (por exemplo, o clima, florestas e rios). Esses processos interagem em múltiplas escalas, do local ao global. Além disso, sistemas socioecológicos exibem propriedades emergentes, não lineares e mudanças imprevisíveis (WALKER; SALT, 2006). Resiliência é a capacidade de um sistema absorver perturbações externas e ainda manter suas propriedades essenciais, incluindo serviços ecossistêmicos essenciais para o bem-estar humano. Resiliência é um conceito muito mais dinâmico do que sustentabilidade porque, em vez de tentar definir algum nível máximo de perturbação que não pode ser ultrapassado, seu foco está na capacidade de mudança (BUSCHBACHER, 2014). Enquanto esses sistemas são muitas vezes manejados para maximizar um número limitado de recursos, baseado em premissas que estes têm funções de produção lineares (HOLLING; MEFFE, 1996), a abordagem de resiliência procura informar a gestão para a capacidade que os sistemas têm de aprender, se auto-organizar e se adaptar em face de mudanças imprevisíveis e não lineares.

Este artigo abre este dossiê sobre a aplicação do pensamento da resiliência como uma ferramenta para a compreensão e, possivelmente, a gestão da fronteira amazônica. Os artigos são baseados nos resultados de um Curso de Especialização em Gestão Socioambiental, que envolveu um grupo multidisciplinar e multinstitucional de profissionais. O curso utilizou conceitos e métodos de resiliência para analisar a dinâmica de sistemas socioecológicos complexos na região noroeste do Mato Grosso na Amazônia brasileira. Os participantes foram motivados a explorar o pensamento e os conceitos de resiliência porque eles foram considerados potencialmente relevantes para as seguintes questões que afetam diretamente o seu trabalho:

- Quais são os fatores críticos na tomada de decisões do uso da terra por diferentes atores sociais, especificamente (i) proprietários de terras médias e grandes que se dedicam à pecuária e exploração madeireira em larga escala, (ii) os povos indígenas, e (iii) pequenos produtores e colonos? Como as decisões individuais afetam o uso da terra, e se acumulam para gerar um comportamento mais amplo no sistema (impactos sociais, econômicos e ambientais)?

- Quais são as principais interações no sistema (entre proprietários de terras, políticas públicas, através de escalas, etc.)? Podemos identificar potenciais pontos de limiar (grandes não linearidades, em que a introdução de pequenas mudanças no sistema (inputs) resulta em grandes e súbitas mudanças ao final (outputs))?

- Como o sistema se auto-organiza, e qual é a sua capacidade de aprendizagem e adaptação? Como essas capacidades de adaptação podem ser aumentadas?

Este artigo apresenta o pensamento de resiliência e sua relevância para o trabalho de profissionais de conservação e desenvolvimento. Também apresenta uma ferramenta de avaliação de resiliência para a aplicação do pensamento de resiliência visando aumentar a compreensão dos sistemas socioecológicos complexos. Essa ferramenta foi incorporada a um Curso de Especialização em Gestão Colaborativa de Sistemas Socioecológicos na Amazônia Brasileira, a partir do qual este dossiê se originou. Os cinco artigos seguintes apresentam estudos de caso de aplicação do pensamento de resiliência em sistemas socioecológicos específicos da Amazônia brasileira. O artigo de encerramento do dossiê (BARTELS et al., 2016) apresenta a pedagogia que foi utilizada para coconstruir um espaço comum de reflexão e aprendizagem sobre resiliência e gestão colaborativa no curso. Bartels descreve os desafios e lições aprendidas com a aplicação da ferramenta de avaliação de resiliência em um processo participativo que incorporou diversos interesses e visões de mundo. Seu artigo também faz uma reflexão sobre a 
aplicabilidade da ferramenta de avaliação de resiliência para a gestão territorial em áreas disputadas da fronteira amazônica.

O presente artigo apresenta o contexto regional e institucional do curso de especialização, discute a ferramenta de avaliação de resiliência que foi desenvolvida e aplicada no curso, e conclui com observações sobre o sistema socioecológico regional que surgiram da avaliação.

\section{CONTEXTO REGIONAL E ORGANIZACIONAL}

O curso de especialização foi desenvolvido em quatro módulos, baseados em trabalho de campo, no município de Cotriguaçu, noroeste de Mato Grosso, Amazônia brasileira (Veja figura 1). Mato Grosso tem expandido rapidamente sua agricultura e pecuária, e desde 2004 foi o estado com as maiores taxas de desmatamento no Brasil (BABY, 2012). No noroeste do estado, uma extensa colonização ocorreu desde a década de 1980, porém, ainda é uma das regiões mais isoladas, onde a infraestrutura é pobre, e há uma grande área de floresta remanescente (81\% em 2008, segundo LENTINI, 2010). Cotriguaçu mistura áreas de exploração madeireira, fazendas, áreas de colonização de pequenos produtores, Terras Indígenas e Áreas Protegidas, representando assim os conflitos e dinâmicas de sistemas socioecológicos típicos de regiões de fronteira amazônica.

Quando o curso começou em 2010, Cotriguaçu tinha sido selecionada como uma área focal para o desenvolvimento de um projeto-piloto de REDD² no Mato Grosso (ICV, 2009). Embora nenhum projeto de REDD tenha acontecido, a ONG regional Instituto Centro de Vida, a Secretaria Estadual de Meio Ambiente e o governo municipal de Cotriguaçu estão colaborando em um grande projeto que visa tornar o uso da terra na região mais sustentável³.

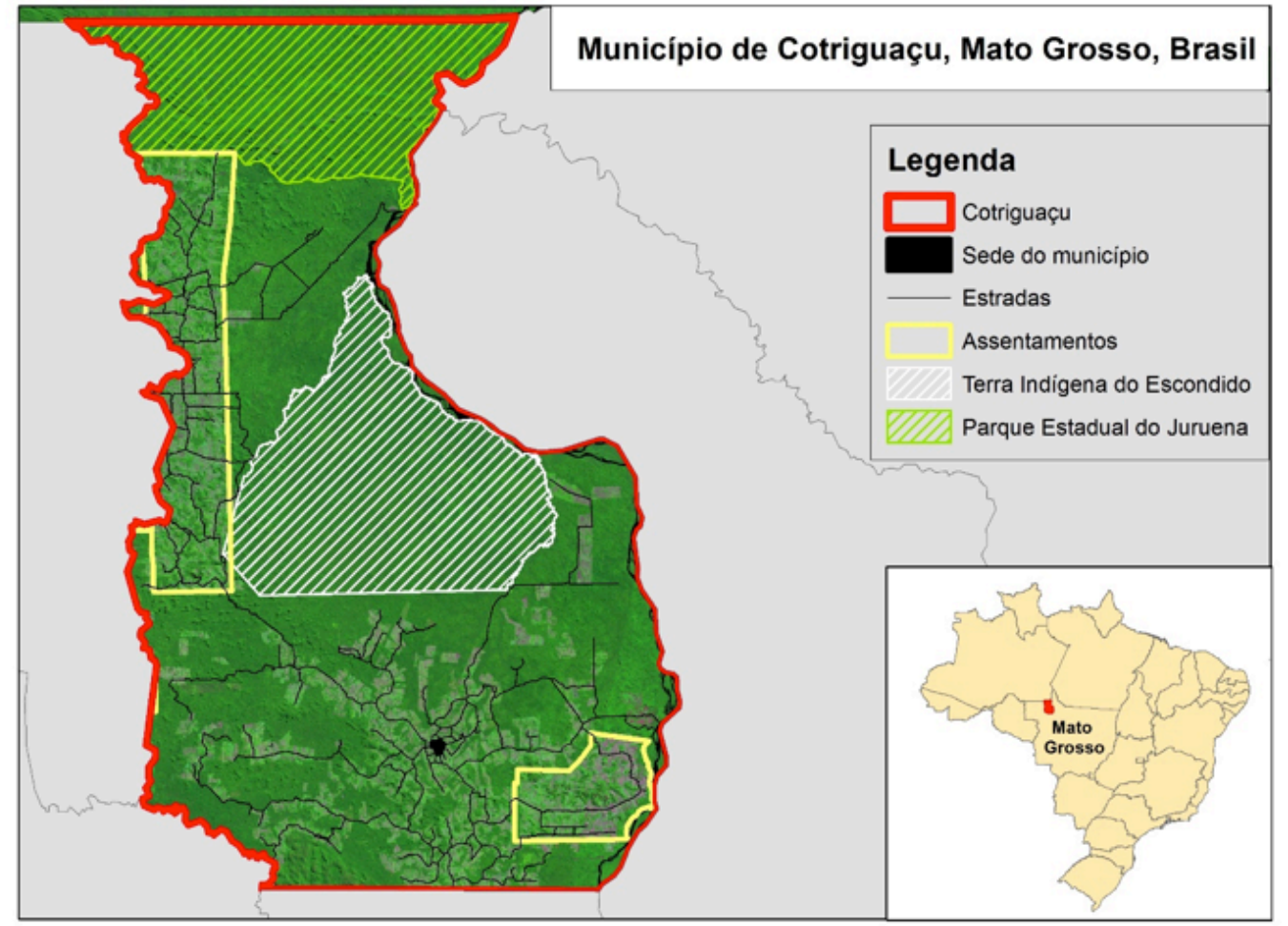

Figura 1 - Mapa do município de Cotriguaçu, noroeste de Mato Grosso, mostrando diferentes categorias de posse da terra e cobertura vegetal. Elaborado por Paula Bernasconi com dados do IBGE, Incra, Sema, Funai.

O curso de especialização, além de abordar simultaneamente temas de colaboração e pedagogia crítica (BARTELS et al., 2016), foi organizado em torno de uma série de exercícios para praticar e testar a avaliação de resiliência apresentada a seguir. Os participantes do curso formaram três grupos e realizaram a avaliação de resiliência para os três principais grupos sociais que manejam diretamente recursos e 
tomam decisões sobre mudanças no uso e cobertura da terra em Cotriguaçu: (i) proprietários de terras médias e grandes que se dedicam à exploração madeireira e pecuária, (ii) os povos indígenas Rikbaktsa, e (iii) agricultores familiares. Esses grupos de participantes trabalharam em paralelo para entender a história, caracterizar os principais atributos e objetivos, e considerar cenários futuros para cada grupo social. Para a fase final da análise, os grupos do curso se reuniram para considerar as interações entre os diferentes grupos sociais do município de Cotriguaçu e como potenciais ações de gestão poderiam promover um futuro positivo para os grupos tanto individual como coletivamente.

Os seguintes artigos do dossiê apresentam os resultados do exercício de avaliação de resiliência para os proprietários de terras médias e grandes de Cotriguaçu (BERNASCONI et al., 2016), e para os povos indígenas Rikbaktsa que habitam Cotriguaçu e outros territórios no noroeste de Mato Grosso (ALMEIDA et al., 2016). Além dos exercícios em grupo, cada participante do curso realizou um projeto individual aplicando conceitos de resiliência ao seu próprio trabalho como profissionais de conservação e desenvolvimento, seja em Cotriguaçu ou em outras comunidades da Amazônia brasileira. Olival (2016) adaptou a metodologia de avaliação de resiliência como uma ferramenta para o planejamento estratégico e operacional do Instituto Ouro Verde, uma organização não governamental que atua apoiando agricultores familiares no norte de Mato Grosso. Simão e Athayde (2016) avaliaram a resiliência das comunidades ribeirinhas tradicionais que sofreram deslocamento forçado devido à construção de uma barragem hidroelétrica em Rondônia, focando na sua capacidade de resistência cultural. Finalmente, Sobreiro (2016) analisou a dinâmica da pesca ornamental no município de Barcelos (AM), focando na evolução histórica do sistema, suas reorganizações e resiliência.

\section{METODOLOGIA PARA AVALIAÇÃO DA RESILIÊNCIA}

A Resilience Alliance é uma rede de pesquisa composta por cientistas e profissionais de diferentes disciplinas que trabalham para desenvolver e aplicar os conceitos da resiliência (www.resalliance.org). A Aliança tem desenvolvido uma metodologia de "Avaliação da Resiliência" como "uma forma alternativa de pensar e praticar a gestão dos recursos naturais", destinada a "profissionais, gestores e outras partes interessadas que têm o desejo ou a capacidade de influenciar as decisões e ações no sistema" (RESILIENCE ALLIANCE, 2007a). Existem vários formatos para a realização de uma avaliação de resiliência, conforme apresentado em duas versões de um manual para profissionais (RESILIENCE ALLIANCE, 2007a; RESILIENCE ALLIANCE, 2010), um manual para cientistas (RESILIENCE ALLIANCE, 2007b) e publicações como Walker et al. (2009) e Strickland-Munro et al. (2010). Essa literatura é bastante consistente em uma abordagem geral: um grupo com conhecimento especializado vai definir os limites do sistema, seus componentes e questões-chave; vai caracterizar dinâmicas reais e potenciais do sistema (limiares e estados alternativos), e usa esse entendimento para decidir "onde e como intervir no sistema, a fim de aumentar a resiliência" (RESILIENCE ALLIANCE, 2007a). No entanto, os manuais e artigos variam consideravelmente em termos de organização, sequência e passos específicos a serem realizados. Além disso, há pouca ou nenhuma orientação sobre como determinar as questões-chave, atributos ou estados desejáveis do sistema, mas sim um pressuposto de que tais determinações devem ser feitas de forma rápida e objetiva (como indicado pela expectativa de que toda a avaliação pode ser realizada em um workshop com duração de cerca de três dias (RESILIENCE ALLIANCE, 2007a)).

Essas limitações se tornaram claras quando tentamos utilizar o Manual de Avaliação da Resiliência para Profissionais (RESILIENCE ALLIANCE, 2007a) como uma diretriz ou "roteiro" para avaliar a resiliência em Cotriguaçu como parte do curso de especialização. Dadas as visões de mundo, perspectivas e epistemologias altamente variáveis trazidas ao curso pelo grupo de participantes diversificado e multidisciplinar, o manual foi contestado, negociado e revisto ao longo do processo. Ele foi aplicado de forma diferenciada por cada grupo de participantes, refletindo suas perspectivas particulares e as diferentes situações dos atores sociais sujeitos do estudo.

Bartels et al. (2016) fornecem uma reflexão aprofundada sobre os desafios e limitações que emergiram durante o curso, e as implicações desses desafios para a utilização da avaliação de resiliência como instrumento para a cogestão adaptativa. Apesar das dificuldades, os participantes foram capazes de desenvolver a sua própria abordagem para realizar a avaliação de resiliência, a qual é apresentada na 
Figura 2 e descrita na próxima seção deste artigo. Essa nova versão da metodologia apresenta vários avanços. Em primeiro lugar, ela reconhece que podem haver diversas perspectivas sobre a definição de questões-chave e limites de um sistema, e permite que estas variem entre os grupos sociais estudados. Em segundo lugar, ela enfatiza a compreensão da história do sistema, e usa a história como a base para a definição dos principais causadores de mudança e interações entre diferentes escalas. Em terceiro lugar, reconhece que a definição dos atributos-chave do sistema influencia a definição de estados desejáveis e indesejáveis do sistema, e atrasa esse passo até que uma análise aprofundada da história do sistema seja realizada. A análise histórica e outros métodos procuram incorporar as perspectivas e valores dos atores locais na definição dos principais atributos e estados desejáveis do sistema. Finalmente, ela usa cenários como a ferramenta fundamental para a proposição de estados desejáveis no futuro e cria o potencial para o uso de cenários para catalisar o diálogo entre grupos sociais com interesses conflitantes.

A próxima seção descreve as etapas de uma avaliação de resiliência e como elas foram realizadas durante o curso de especialização em Cotriguaçu, seguindo a estrutura apresentada na Figura 2.

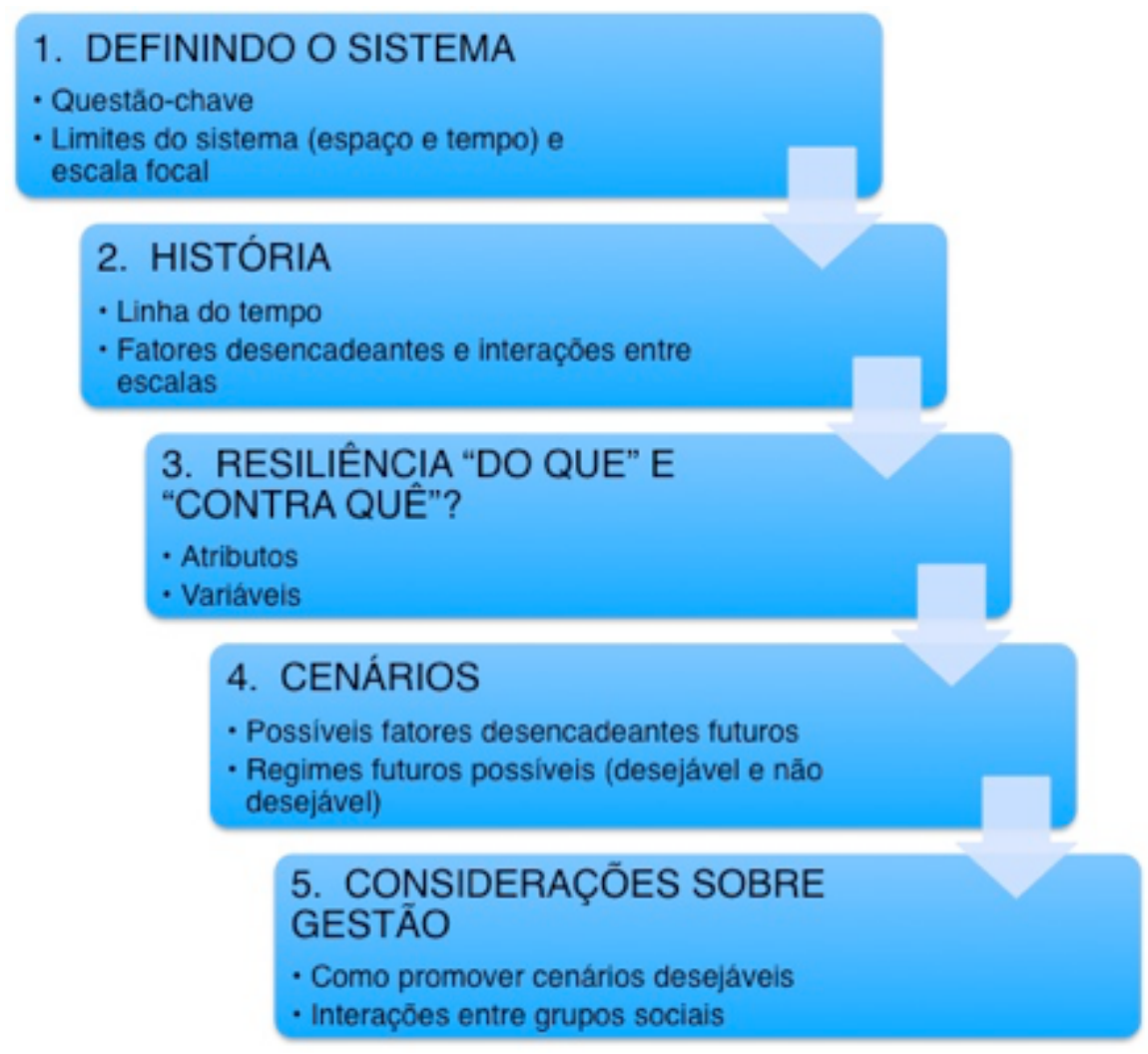

Figura 2 - Metodologia de avaliação da resiliência desenvolvida pelos autores, adaptada de Resilience Alliance 2007a.

\section{DEFINIÇÃO DO SISTEMA}

\section{a. Definição da Questão-Chave}

A avaliação da resiliência começa com a definição de uma "questão-chave". Em Cotriguaçu, a questão foi definida desta forma: Como manter a sustentabilidade ecológica, econômica e social dos modos de vida de cada grupo de atores sociais? Essa questão foi centrada sobre os grupos sociais identificados e foca em como manter a sua presença e sua viabilidade na região. Embora o uso da terra e os impactos ambientais tenham sido explicitamente incluídos na análise, o exercício foi muito mais orientado para o aspecto social do que é comum em grande parte da literatura de resiliência (por exemplo, WALKER et al., 2009). Como apresentado nos artigos seguintes, essa ênfase é consistente com a visão de mundo dos próprios atores sociais em estudo, que enfatizam objetivos sociais e econômicos acima de preocupações ecológicas. 


\section{b. Os limites do sistema e escala focal}

Barnes e Child (2014) sugeriram que "meso" escalas desempenham um papel fundamental na mediação da dinâmica de sistemas socioecológicos, ligando e integrando processos por meio de diferentes escalas. Por exemplo, na Amazônia brasileira atores e organizações municipais (escala meso) estão emergindo como importantes mediadores de governança entre as políticas governamentais estaduais e nacionais (escala macro) e as decisões dos proprietários de terras e produtores rurais (escala micro). Mudanças políticas recentes associadas ao sucesso na redução do desmatamento do Brasil devolveram grande parte da autoridade e responsabilidade da gestão ambiental para os governos municipais (NEVES, 2012). Para os municípios com alta taxa de desmatamento, incluindo Cotriguaçu, restrições de crédito e de licenciamento foram postas em prática em 2006, o que exigiu avanços significativos na gestão ambiental para que essas restrições fossem revogadas (MAIA et al., 2011).

A escala espacial foco deste exercício foi definida como o nível municipal. A escala micro, por sua vez, foi definida como famílias individuais, empresas, fazendas ou propriedades rurais. A escala macro referiu-se a processos estaduais, nacionais ou globais, tais como políticas públicas e mercados.

A escala temporal de análise foi definida com base na história de Cotriguaçu e a dinâmica temporal dos processos de colonização e de uso da terra que foram estudados. A análise histórica se iniciou nos anos 1980. Os exercícios com cenários olharam para um horizonte de 10-20 anos no futuro, a fim de ser relevante para a realidade atual dos grupos sociais e seus potenciais horizontes de planejamento e de investimento. Tal como descrito no artigo de Almeida et al. (2016), essas datas foram modificadas pelo grupo de estudo com foco nos povos indígenas a fim de refletir as dinâmicas históricas específicas desse grupo social.

\section{HISTÓRIA}

\section{a. Linha do tempo: aprendendo sobre a história dos atores sociais}

A análise de cada sistema socioecológico começou com a criação de uma linha do tempo que descreveu a história de um grupo social desde a sua chegada a Cotriguaçu, com base em interações formais e informais com uma amostra dos atores em cada grupo. Essas visitas e entrevistas foram realizadas com atores em suas propriedades rurais, serrarias, assentamentos de reforma agrária e territórios indígenas. O curso também incluiu reuniões com informantes-chave do governo municipal que representam os interesses e prestam serviços a esses diferentes grupos sociais. As informações das entrevistas estruturadas e semiestruturadas foram integradas com o conhecimento dos participantes do curso, baseadas em sua experiência de trabalho com esses grupos sociais.

Linhas do tempo que foram produzidas neste processo, tal como apresentadas nos seguintes artigos deste dossiê, foram interpretadas e apresentadas com base no modelo heurístico de Ciclo de Renovação Adaptativo de Holling e Gunderson (2002; ver também BUSCHBACHER, 2014), dividido em fases de exploração-colonização-liberação-reorganização. Esse modelo mostra alterações que um determinado sistema sofre ao longo do tempo, como uma série de ciclos de adaptação, e também indica se as mudanças foram graduais ou repentinas. O modelo foca em momentos-chave em que o sistema sofre uma grande mudança, conceituada como liberação e subsequente reorganização.

Além da linha do tempo documentar as mudanças temporais no sistema socioecológico, essa etapa da avaliação foi importante para a compreensão de como os atores foram afetados e como eles responderam, de forma a ter uma visão sobre os seus interesses, objetivos e preocupações. As interações dos participantes do curso com os atores locais, e o processo de estudar a história de cada grupo social foram um importante ponto de partida para a compreensão das diferentes perspectivas dos atores e, portanto, relevante para a definição dos principais atributos que caracterizam o sistema (etapa 3 da avaliação), bem como os regimes desejáveis para o exercício dos cenários futuros (etapa 4). 


\section{b. Fatores desencadeantes e interações entre escalas}

A análise histórica mostrou como o sistema mudou ao longo do tempo, e isso tornou possível identificar as principais causas de mudanças (fatores desencadeantes ou drivers) internas e externas, que têm sido prevalentes no passado para cada grupo social. Esses drivers ajudam a entender as interações entre a escala focal (um dado grupo social no município de Cotriguaçu, por exemplo) e as escalas hierárquicas macro e micro que são aninhadas. Com base nessa análise, complementada por discussões com os atores sobre as suas preocupações e expectativas para o futuro, prováveis cenários futuros do sistema foram gerados no exercício da etapa 4.

\section{RESILIÊNCIA DO QUE E CONTRA O QUÊ?}

Esta etapa é conhecida como a definição de "Resiliência do que e contra o quê?", porque um conjunto de "atributos" são escolhidos para caracterizar a essência do sistema, e assim podem ser usados para descrever seu estado em qualquer ponto no tempo (resiliência do que), enquanto as causas (drivers) indicam as forças principais que afetam o sistema e podem causar mudança no sistema (resiliência contra o quê) ${ }^{4}$ (CARPENTER et al., 2001).

\section{a. Atributos}

Os atributos são algumas poucas características que descrevem o estado do sistema. Embora os atributos sejam apresentados no Manual de Avaliação de Resiliência (RESILIENCE ALLIANCE, 2007a) como uma descrição objetiva e de valores neutros, os atributos explicitamente enquadram qualquer discussão de um estado desejável do sistema (GOFFMAN, 1974) e podem, portanto, incorporar perspectivas culturais, éticas e epistemológicas do profissional que o utiliza. Nós definimos os atributos para cada grupo social que analisamos a partir de uma perspectiva êmica, isto é, como os próprios atores representariam as principais características de um estado desejável ou indesejável do sistema. Os atributos foram, portanto, os indicadores da persistência dos grupos sociais, em relação às suas características identitárias desejáveis. Por exemplo, um sistema produtivo diversificado foi considerado um atributochave para o agricultor familiar, ao passo que os proprietários de terras médias e grandes priorizaram viabilidade econômica de sua propriedade em vez de diversificação da produção. A nossa "questãochave" foi definida em torno da manutenção de cada grupo social e sua estratégia de modos de vida, por isso a definição dos atributos foi integralmente relacionada com questões de identidade, tais como a língua e a cultura do grupo indígena.

Atributos foram definidos por meio de um intenso processo de discussão em grupo durante vários módulos do curso e comunicação on-line entre os módulos, e foram baseados tanto na compreensão dos autores em relação a perspectiva e valores dos atores estudados quanto na abordagem teórica e epistemológica adotada pelos autores. Por ser de extrema importância a lente utilizada para analisar o sistema, uma quantidade considerável de discussão e debate ocorreu para a definição dos atributos em cada estudo de caso.

\section{b. Variáveis}

Um passo importante na definição de atributos é operacionalizá-los, definindo indicadores ou variáveis que possam ser mensuradas. Isso foi necessário para ir além de dimensões ou temas gerais (por exemplo, trabalho ou imagem) para ser explícito sobre como o estado desse atributo pode ser medido. Por exemplo, o grupo da agricultura familiar (OLIVAL, 2016) operacionalizou "trabalho" como a proporção do trabalho de uma propriedade rural que é baseada no salário, enquanto o grupo de médios e grandes proprietários (BERNASCONI et al., 2016) operacionalizou o "perfil" desse grupo social, como o grau em que fazendeiros da Amazônia são "vilanizados" pela mídia. É o estado do atributo, isto é, o nível da variável, que é necessário para caracterizar o estado do sistema. A definição de variáveis e a operacionalização dos atributos fizeram parte da discussão em grupo e foram necessárias para que os atributos possam ser aplicáveis na definição do estado do sistema. Como este trabalho foi um exercício dentro de um processo de aprendizagem e não envolveu um grande esforço de coleta de dados, os autores utilizam termos relativos para caracterizar as variáveis, ou seja, alta/média/baixa ou aumentando/diminuindo. 


\section{ANÁLISE DE CENÁRIOS}

Enquanto os passos 1 a 3 da avaliação de resiliência visam caracterizar o sistema e entender suas dinâmicas históricas e atuais, as etapas 4 e 5 olham para o futuro e são destinadas a orientar ações de gestão. Essa fase começa com a análise de cenários, que é uma ferramenta para gerar hipóteses sobre o futuro, sobre como seriam as mudanças no sistema (PETERSON et al., 2003; VAN DER HEIJDEN, 2005). A análise de cenários pode ser utilizada como uma ferramenta para revisar e integrar a avaliação de resiliência. Ela destaca as principais conexões, conflitos, sinergias e compensações entre as "partes" do sistema, dentro de uma paisagem com diversos atores sociais. Os cenários foram desenvolvidos como um exercício facilitado durante módulos do curso, inicialmente com foco em cada um dos três grupos sociais importantes para Cotriguaçu. Os três conjuntos de cenários foram, em seguida, utilizados em conjunto nas etapas finais de avaliação de resiliência, como um ponto de partida para explorar as interações entre os grupos sociais, e para discutir cenários que seriam desejáveis para todos os grupos.

\section{a. Prováveis causas de mudança (drivers) no futuro}

A análise de cenário começou com uma revisão das principais discussões das etapas anteriores na avaliação de resiliência: história, causas de mudança e os atributos selecionados para definir o estado do sistema. A análise histórica e a de mudanças passadas foram combinadas com o conhecimento dos participantes do curso sobre a dinâmica atual da região amazônica para identificar prováveis drivers de grande mudança do sistema, em múltiplas escalas, nas próximas duas décadas. Isso inclui mudanças em políticas públicas, a dinâmica do mercado, desenvolvimento de projetos de infraestrutura e tendências observadas e projetadas na qualidade e quantidade dos recursos naturais.

\section{b. Regimes futuros possíveis}

A questão que a análise de cenários traz à tona é: como possíveis drivers afetarão os atributos do sistema no futuro? De uma perspectiva de resiliência, o conjunto atual de atributos se mantém no mesmo regime (sistema resiliente), ou haverá uma mudança fundamental do regime (transformação)? É importante reconhecer que a análise de cenários não é uma tentativa de prever o futuro, mas, sim, uma ferramenta para visualizar diferentes possíveis trajetórias do sistema em termos de histórias plausíveis e consistentes (RASKIN et al., 2005; VAN DER HEIJDEN, 2005). Portanto, os cenários positivos e negativos foram criados para cada grupo social postulando mecanismos pelos quais os potenciais drivers poderiam levar a estados desejáveis ou indesejáveis dos atributos do sistema. Cada um desses cenários consistiu de:

i) uma descrição geral do regime futuro hipotético do sistema;

ii) o estado projetado das variáveis para cada um dos atributos definidos na etapa 3 da avaliação de resiliência para esse regime;

iii) uma narrativa - expressa tanto textual como artisticamente por meio de desenhos ou dramatização - contando como o sistema poderia passar de condições atuais a esse possível futuro regime.

Cenários para cada grupo incluíram a consideração de como um determinado cenário poderia afetar, ou ser afetado pela dinâmica dos outros grupos sociais no sistema. Por exemplo, um cenário em que as mudanças de política do governo permitissem que grandes proprietários pudessem explorar madeira em Terras Indígenas, aumentaria as oportunidades econômicas para o setor, aumentaria a oferta de oportunidades de trabalho remunerado fora da exploração agrícola para os agricultores (que muitas vezes trabalham em serrarias), porém, reduziria a integridade territorial da comunidade indígena.

\section{CONSIDERAÇÕES SOBRE GESTÃO}

A avaliação de resiliência é apresentada nos manuais da Resilience Alliance como um processo que termina em uma série de ações de manejo. Como explicado acima, os estudos de caso apresentados neste dossiê não estavam envolvidos diretamente em processos de gestão, mas foram realizados como 
um exercício para aprender sobre o sistema socioecológico Cotriguaçu e sobre a metodologia de resiliência. No entanto, nessa etapa final, o exercício de cenários foi utilizado para catalisar a reflexão e discussão entre os membros do grupo sobre como as conclusões de uma avaliação da resiliência podem ser usadas para promover cenários desejáveis. Isso foi feito em cada um dos três grupos sociais separadamente (proprietários de terras médias e grandes, a comunidade indígena, agricultura familiar) e, em seguida, olhando para todo o conjunto de grupos sociais e como eles interagem.

\section{a. Como promover um cenário desejável?}

Com base nos possíveis futuros drivers e regimes que emergiram da análise de cenário na etapa 4, e levando em consideração as possíveis interações negativas ou positivas entre grupos sociais, participantes foram incumbidos de identificar passos para: mover o sistema para uma direção percebida como positiva; preparar-se para possíveis riscos, mudanças e cenários alternativos; e promover resiliência geral em termos de aprendizagem, capacidade, flexibilidade, etc.

\section{b. Interações entre os grupos sociais}

Até este ponto, três avaliações de resiliência foram realizadas em paralelo, uma para cada grupo social. Nessa etapa final, o foco mudou para como os diferentes grupos sociais interagem uns com os outros e, especificamente, sobre a forma como o cenário desejável de um grupo social afetaria outros grupos sociais. Representantes de cada grupo apresentaram suas ações propostas para aumentar a resiliência do seu próprio grupo a cada um dos outros grupos. Cada grupo então avaliou como os cenários desejáveis e ações propostas dos outros grupos os afetaria positiva ou negativamente (como no exemplo acima sobre a exploração madeireira no território indígena). A partir da compreensão dessas interações, cada grupo social poderia modificar seus cenários desejáveis e estratégias propostas, a fim de ter uma interação mais positiva com os outros setores, ou pode decidir envolver-se em competição e conflito, a fim de colocar seus próprios interesses acima dos outros grupos.

\section{OBSERVAÇÕES GERAIS SOBRE O SISTEMA SOCIOECOLÓGICO COTRIGUAÇU QUE EMERGIRAM DO EXERCÍCIO DE AVALIAÇÃO DE RESILIÊNCIA}

Os exercícios de avaliação de resiliência, baseados em observações dos três grandes grupos sociais de Cotriguaçu, forneceram informações gerais sobre o sistema socioecológico regional. Conclusões gerais para cada um dos três grupos sociais e a sua interação no sistema focal integrado de Cotriguaçu são apresentadas abaixo.

\section{HISTÓRIA}

A análise histórica revelou que cada grupo social tem passado por uma série de mudanças que se encaixam bem no modelo heurístico do ciclo adaptativo. A Tabela 1 apresenta os principais ciclos históricos identificados para cada grupo. Dois ciclos completos foram claramente identificados para os proprietários de terras médias e grandes, com base nos modelos de produção predominantes (inicialmente a agricultura com pouca exploração madeireira, seguida de pecuária com exploração madeireira extensiva). Um terceiro ciclo está agora em curso, com uma tendência, ainda não consolidada, para sistemas de produção mais sustentáveis na pecuária. 
Tabela 1 - Ciclos e fatores desencadeadores de mudança a partir da análise histórica, e possíveis desencadeadores futuros identificados no exercício de cenários, para três grupos sociais no norte de Mato Grosso, Brasil.

\begin{tabular}{|c|c|c|c|}
\hline Grupo Social & Principais ciclos históricos & $\begin{array}{l}\text { Principais desencadeadores de } \\
\text { mudança }\end{array}$ & $\begin{array}{l}\text { Potenciais desencadeadores } \\
\text { futuros }\end{array}$ \\
\hline \multirow[t]{3}{*}{$\begin{array}{l}\text { Proprietários de } \\
\text { terras médias e } \\
\text { grandes } \\
\text { (BERNASCONI et } \\
\text { al., 2016) }\end{array}$} & $\begin{array}{l}\text { Colonização; agricultura e } \\
\text { alguma extração madeireira } \\
(1974 \text { - 1990) }\end{array}$ & $\begin{array}{l}\text { Políticas de colonização da Amazônia } \\
\text { combinadas com imigração vinda do } \\
\text { Sul do Brasil; Ajustamento estrutural e } \\
\text { reformas políticas neoliberais levando } \\
\text { ao colapso da Cooperativa }\end{array}$ & \multirow{3}{*}{$\begin{array}{ll}\text { Positivos: } \\
\square \quad \begin{array}{l}\text { Demanda global por } \\
\text { alimentos }\end{array} \\
\square \quad \text { Pavimentação de estradas } \\
\text { Negativos: } \\
\square \quad \begin{array}{l}\text { Competição global por } \\
\text { fornecimento de alimentos }\end{array} \\
\square \quad \begin{array}{l}\text { Aumento da fiscalização e } \\
\text { controle ambiental }\end{array}\end{array}$} \\
\hline & $\begin{array}{l}\text { Pecuária e extração } \\
\text { madeireira extensiva } \\
(1990-2006)\end{array}$ & $\begin{array}{l}\text { Preocupações ambientais globais sobre } \\
\text { o aumento do desmatamento da } \\
\text { Amazônia; Operações de fiscalização } \\
\text { ambiental contra o desmatamento e } \\
\text { exploracão madeireira ilegais }\end{array}$ & \\
\hline & $\begin{array}{l}\text { Reorganização da pecuária e } \\
\text { extração madeireira para } \\
\text { práticas sustentáveis } \\
\text { (2006-presente) }\end{array}$ & $\begin{array}{l}\text { Descentralização do licenciamento } \\
\text { florestal; Políticas de controle de } \\
\text { desmatamento; Infestação das } \\
\text { pastagens com cigarrinha }\end{array}$ & \\
\hline \multirow{5}{*}{$\begin{array}{l}\text { Povos indígenas } \\
\text { Rikbaktsa } \\
\text { (ALMEIDA et al., } \\
\text { 2016) }\end{array}$} & Tempos imemoriais & & \multirow{5}{*}{$\begin{array}{l}\text { Usinas hidrelétricas } \\
\text { Possível enfraquecimento dos } \\
\text { direitos legais } \\
\text { Possível divisão do município de } \\
\text { Cotriguaçu }\end{array}$} \\
\hline & $\begin{array}{l}\text { Colonização do Vale do Rio } \\
\text { Juruena }\end{array}$ & $\begin{array}{l}\text { Epidemias; } \\
\text { Colonização por seringueiros }\end{array}$ & \\
\hline & $\begin{array}{l}\text { Pacificacão missionária } \\
\text { (Década de 1950) }\end{array}$ & $\begin{array}{l}\text { Missão Anchieta; Transferência de } \\
\text { crianças e jovens indígenas para o } \\
\text { Internato Utiariti }\end{array}$ & \\
\hline & $\begin{array}{l}\text { Regularização fundiária e } \\
\text { reorganização sociopolítica } \\
(1970-1990)\end{array}$ & $\begin{array}{l}\text { Retorno das crianças do Internato } \\
\text { missionário; } \\
\text { Demarcação das Terras Indígenas }\end{array}$ & \\
\hline & $\begin{array}{l}\text { Conflitos territoriais (2000- } \\
\text { presente) }\end{array}$ & $\begin{array}{l}\text { Expansão do agronegócio e } \\
\text { infraestrutura }\end{array}$ & \\
\hline \multirow[t]{8}{*}{$\begin{array}{l}\text { Agricultores } \\
\text { familiares } \\
\text { (OLIVAL, 2016) }\end{array}$} & Período anterior à colonização & $\begin{array}{l}\text { História de outras regiões do Brasil; } \\
\text { Modelo de desenvolvimento } \\
\text { predominante (capitalismo global) }\end{array}$ & \multirow{8}{*}{$\begin{array}{l}\text { Aumento do controle ambiental } \\
\text { Políticas públicas favorecendo } \\
\text { produção em grande escala }\end{array}$} \\
\hline & $\begin{array}{l}\text { Colonização (Década de } \\
\text { 1970) }\end{array}$ & $\begin{array}{l}\text { Colonização privada } v s \text { colonização } \\
\text { pública }\end{array}$ & \\
\hline & $\begin{array}{l}\text { Desenvolvimento e } \\
\text { decadência de infraestrutura } \\
\text { comunitária }\end{array}$ & $\begin{array}{l}\text { Programas do Instituto Nacional de } \\
\text { Colonização e Reforma Agrária } \\
\text { (Incra); } \\
\text { Programas de Eletrificação Rural }\end{array}$ & \\
\hline & $\begin{array}{l}\text { Desenvolvimento e declínio } \\
\text { da organização comunitária }\end{array}$ & $\begin{array}{l}\text { Dinâmica social dentro das } \\
\text { comunidades }\end{array}$ & \\
\hline & $\begin{array}{l}\text { A evolução dos sistemas de } \\
\text { produção da agricultura para a } \\
\text { pecuária }\end{array}$ & $\begin{array}{l}\text { Clima; } \\
\text { Preços das commodities; } \\
\text { Dificuldades de mercado; } \\
\text { Programas de crédito do governo } \\
\text { favorecendo a pecuária }\end{array}$ & \\
\hline & $\begin{array}{l}\text { Mineração (Décadas 1980- } \\
\text { 1990) }\end{array}$ & $\begin{array}{l}\text { Descoberta de ouro e seus altos preços } \\
\text { de mercado }\end{array}$ & \\
\hline & $\begin{array}{l}\text { Apoio à produção e } \\
\text { comercialização (desde 2004) }\end{array}$ & $\begin{array}{l}\text { Programa Nacional de Aquisição de } \\
\text { Alimentos da Agricultura Familiar } \\
\text { (PAA); } \\
\text { Projetos de ONGs }\end{array}$ & \\
\hline & Novos desafios de produção & $\begin{array}{l}\text { Infestação cigarrinha; } \\
\text { Síndrome da morte súbita do pasto; } \\
\text { Escassez de água }\end{array}$ & \\
\hline
\end{tabular}

O processo de colonização e assentamento do grupo agricultura familiar é marcado por crescimento e declínio da infraestrutura e organização comunitária, e uma mudança dos sistemas de produção da agricultura diversificada para uma ênfase em pecuária. A dinâmica desse sistema foi fortemente marcada por um boom de mineração de ouro na região, que despovoou as propriedades, causando um aumento na imigração e degradação ambiental.

O grupo indígena Rikbaktsa tem uma história muito mais antiga na região do que outros grupos, reconhecida como enraizada nos "tempos imemoriais". Esse sistema tem sofrido uma série de reorganizações, causadas por desterritorialização, a pacificação missionária, e reterritorialização dos indígenas em uma pequena parte de seu território original. A história em curso é de luta contínua pela manuten- 
ção do território e direitos, desenvolvimento para satisfazer suas necessidades básicas, e autodeterminação cultural. Enquanto os grupos sociais agricultores familiares e proprietários de terras médias e grandes identificam o início de sua história na região com a colonização na década de 1980, ambos também reconhecem que a "pré-história" de seus grupos em suas regiões de origem é essencial para a compreensão da dinâmica dos grupos em Mato Grosso.

Quando avaliamos os três grupos juntos, podemos ver o quão dinâmico é o sistema, com mudanças dramáticas nas formas de produção, uso do solo e controle territorial. No entanto, os proprietários de terras médias e grandes têm persistentemente mantido posições econômicas, políticas e culturais dominantes. Eles têm mudado entre a agricultura, exploração florestal e pecuária, mantendo ou expandindo seu controle territorial, com altos níveis de produção econômica e poder político. Os Rikbaktsa, por outro lado, têm passado por processos que ameaçam sua própria existência. A agricultura familiar tem mantido a sua presença na região, mas ainda precisa consolidar um modelo socioeconômico e de produção que lhes garanta uma melhor qualidade de vida; sua persistência e desenvolvimento econômico são, portanto, incertos.

\section{FATORES DESENCADEANTES E INTERAÇÕES ENTRE ESCALAS}

A revisão das principais causas das mudanças históricas nesse sistema (Tabela 1) chama a atenção para o quanto a dinâmica do sistema local para cada grupo social é determinada por fatores de uma escala maior, por exemplo decisões ou processos relacionados às políticas nacionais, mudança no uso da terra no Sul do Brasil ou aos mercados globais.

Os Rikbaktsa foram influenciados por ondas de imigração, desde os primeiros exploradores (Bandeirantes), que trouxeram doenças epidêmicas, e também seringueiros, missionários e colonos que invadiram seu território ancestral. O governo federal tem desempenhado um papel impactante, promovendo e apoiando cada uma dessas ondas de imigrantes, mas também fornecendo direitos e demarcação de porções limitadas de território Rikbaktsa.

Agricultores familiares e proprietários de terras médias e grandes chegaram à região movidos por políticas nacionais de desenvolvimento e dinâmicas de uso da terra em outras partes do Brasil. A história de ambos os grupos foi influenciada por políticas governamentais relacionadas à infraestrutura, incentivos econômicos e regulamentação ambiental. Por exemplo, a Cooperativa Agrícola de Cotriguaçu falhou em 1990 devido a mudanças nas políticas agrícolas federais, e sua indústria madeireira foi fechada devido a operações de fiscalização ambiental. Por outro lado, a chegada de estradas, eletricidade e telefonia celular, por meio de políticas estaduais e federais, teve um grande impacto sobre a qualidade de vida nessas comunidades.

Essa observação não nega a agência dos atores locais. O sistema é fortemente condicionado por políticas e forças externas, e ciclos de colapso e reorganização têm sido impulsionados por eventos em escalas maiores. No entanto, a história da região é determinada pela forma como os atores locais organizam suas fazendas e comunidades, aprendem e adaptam-se a essas forças motrizes. Proprietários de terras médias e grandes não só adaptaram seus sistemas de produção em resposta às políticas vigentes e oportunidades econômicas, mas também se organizaram internamente e com lobistas e grupos políticos em escalas estaduais e nacionais para influenciar as políticas econômicas e ambientais. Os grupos indígenas e da agricultura familiar também têm se adaptado às forças econômicas e de mercado, por exemplo a conversão da agricultura para a pecuária, ou a produção de castanha do Brasil para os mercados nacionais e globais. Esses grupos também se organizam interna e externamente, por meio de alianças com ONGs e organizações nacionais, para resistir às políticas governamentais negativas ou demandar o reconhecimento de seus direitos. No entanto, essa agência local é muitas vezes reativa e geralmente tenta influenciar fatores que vêm de uma escala maior do que as suas próprias comunidades ou região. 


\section{ATRIBUTOS}

Os atributos principais que foram considerados para caracterizar o estado do sistema socioeconômico de cada grupo social foram definidos a partir de uma perspectiva êmica, tendo em conta a perspectiva dos membros desse grupo social. No entanto, a definição dos atributos é muito mais uma interpretação feita pelos grupos de investigação, com base em rodadas de entrevistas (para os grupos indígenas e proprietários de terras médias e grandes), ou definido em um processo participativo entre líderes comunitários e agentes de extensão (para agricultores familiares). A definição dos atributos foi um desafio porque, como em qualquer representação etnográfica, ela é influenciada pela formação dos pesquisadores e é limitada pelo alcance de sua análise (por causa do tamanho e representatividade da amostra, barreiras de comunicação, etc.). Foi útil centrar a análise inicialmente no histórico dos atores da região porque identificou momentos-chave e mudanças, o que auxiliou no entendimento dos fatores e processos na região.

A Tabela 2 apresenta os principais atributos que foram identificados para cada grupo social, organizados em três grandes categorias comuns aos grupos sociais estudados: o acesso à terra e recursos, economia e organização social. Esses atributos representam as principais preocupações e interesses de cada grupo social, os fatores que eles priorizam ao avaliar o estado do sistema e como desejam que o sistema esteja.

Tabela 2 - Atributos identificados em relação aos três grupos sociais no norte de Mato Grosso, Brasil, organizados por categoria.

\begin{tabular}{|c|c|c|c|}
\hline Categorias de atributos & $\begin{array}{c}\text { Proprietários de terras } \\
\text { médias e grandes }\end{array}$ & Povos indígenas Rikbaktsa & Agricultores familiares \\
\hline $\begin{array}{c}\text { Direitos de propriedade e } \\
\text { acesso a recursos }\end{array}$ & Controle da terra & Soberania territorial & Relação com a terra \\
\hline $\begin{array}{c}\text { Renda e aspectos } \\
\text { econômicos }\end{array}$ & Viabilidade econômica & $\begin{array}{c}\text { [Consequência da soberania } \\
\text { territorial] }\end{array}$ & $\begin{array}{c}\text { Uso do trabalho familiar } \\
\text { Sistema de produção }\end{array}$ \\
\hline Organização social & $\begin{array}{c}\text { Organização social } \\
\text { Perfil público }\end{array}$ & $\begin{array}{c}\text { Autodeterminação } \\
\text { sociocultural }\end{array}$ & Organização social \\
& & & \\
\hline
\end{tabular}

Os atributos "controle sobre a terra" para médios e grandes proprietários e "soberania territorial" para indígenas significam acesso a recursos-chave. Para o grupo indígena não foi identificado um atributo econômico específico, porque o desenvolvimento econômico foi considerado uma consequência da soberania sobre sua terra. Em contraste, para o grupo agricultura familiar, o atributo foi a "natureza da relação com a terra", isto é, se o agricultor percebe sua terra como um investimento (perfil capitalista) ou como um meio de reprodução social (perfil camponês). Da mesma forma, os atributos econômicos relacionados à agricultura familiar foram a diversificação da produção e utilização de mão de obra familiar, enquanto que os atributos econômicos identificados para os médios e grandes proprietários foram maximização da renda e viabilidade econômica.

A Organização Social foi considerada um atributo importante para todos os atores sociais. A cooperação foi considerada importante para sua viabilidade e qualidade de vida, tanto pela capacidade de influenciar as políticas públicas quanto pela sua importância social. Além disso, foram identificadas preocupações em todos os grupos sobre identidade. Para o grupo indígena identidade relacionada a sua autodeterminação cultural e linguística, e para os médios e grandes proprietários preocupações sobre como eles são retratados pela mídia e percebidos pelo público.

Notavelmente, fatores ambientais não foram identificados como atributos-chave por nenhum dos grupos. O meio ambiente é visto como uma fonte de recursos, como uma ameaça em termos de regulamentação ambiental, ou como um componente da qualidade de vida. Porém não foi citado pelos atores como uma característica essencial para seu grupo ou para seu modo de vida. 


\section{INTERAÇÕES ENTRE OS GRUPOS SOCIAIS}

A predominância de interações entre diferentes escalas, com destaque da influência de maiores escalas sobre cada grupo social localmente, acabou não evidenciando as interações entre os três grupos sociais durante a maior parte da análise. $\mathrm{O}$ grupo indígena foi uma exceção, porque vem sendo negativamente afetado pela colonização não indígena na região. $O$ conflito fundiário entre agricultores familiares e proprietários de terras médias e grandes é menos prevalente, porque suas terras estão regularizadas (ao contrário das terras devolutas em outras regiões de fronteira na Amazônia). No entanto, existe um conflito entre indígenas e médio e grandes produtores: a demarcação da terra indígena em Cotriguaçu ocorreu em 1998, após a venda dessas terras por uma empresa de colonização. As empresas madeireiras querem explorar a madeira dentro da terra indígena, que é percebida por estas como subutilizada, e há relatos de roubo de madeira e invasão de terras. Outras interações entre as comunidades de agricultura familiar e empresas madeireiras incluem a oferta e a procura de trabalho, assim como a compra de madeira e construção de estradas secundárias em assentamentos agrícolas.

O exercício de cenários trouxe os conflitos entre os grupos de atores sociais à tona. Um cenário positivo para os proprietários de terras médias e grandes inclui a supressão da terra indígena, bem como um maior desenvolvimento da infraestrutura do município, enquanto o cenário negativo para o grupo indígena gira em torno de enfraquecimento de sua soberania territorial e construção de barragens hidroelétricas. Embora tenha havido conflito de pequena escala entre os assentamentos de agricultura familiar e grupos indígenas, os cenários não mostram quaisquer conflitos inerentes ou essenciais entre esses grupos, e ambos poderiam se beneficiar de políticas públicas que favoreçam sistemas de produção diversificados em pequena escala. Em contraste, enquanto existe potencial para a coexistência pacífica, não existe um cenário comum considerado ótimo para ambos proprietários de terras médias e grandes e indígenas.

\section{CONCLUSÕES}

Este artigo propõe uma estrutura analítica para a caracterização de um sistema socioecológico complexo em termos de sua dinâmica histórica e atributos-chave, incluindo os fatores desencadeantes de mudanças e as interações entre diferentes escalas e grupos sociais. Ele avança os métodos de avaliação de resiliência baseando sua análise do sistema na trajetória histórica, buscando incorporar explicitamente as perspectivas dos atores locais, e usando exercícios de cenários para desenvolver possíveis intervenções para gestão.

Nossa análise não conseguiu quantificar a resiliência. Não obstante, ela chamou atenção para a forma como o sistema mudou, como essas mudanças afetaram os atores e, por meio do exercício de cenários, identificou as ameaças e oportunidades para mudanças futuras. Os participantes do curso de especialização consideraram que a ferramenta de avaliação de resiliência forneceu uma perspectiva única e útil sobre o sistema, mesmo que se mostrou de difícil aplicação. Tal aprendizagem pode contribuir para antecipação de surpresas, adaptação e, portanto, a resiliência geral de sistemas socioecológicos.

\section{NOTAS}

${ }^{1}$ No caso das Áreas Protegidas, 39.8\% foram criadas entre 2003 e 2006.

2 “REDD (Redução das Emissões por Desmatamento e Degradação florestal/Reducing Emissions from Deforestation and Forest Degradation) é um esforço para criar um valor financeiro para o carbono armazenado nas florestas, oferecendo incentivos para que os países em desenvolvimento reduzam as emissões florestais e para que invistam em projetos de baixo carbono para o desenvolvimento sustentável “. http://www.un-redd.org/ aboutredd. Acessado em 18 de fevereiro de 2016.

${ }^{3}$ http://www.icv.org.br/como_atuamos/cotriguacu_sempre_verde/ Acessado em 7 de maio de 2015.

${ }^{4} \mathrm{O}$ "estado" do sistema é o conjunto de valores de diferentes atributos em um determinado ponto no tempo. A 
natureza dinâmica dos sistemas significa que o estado está em constante mudança, movendo-se através de um "espaço de estados." Um regime é uma porção do espaço de estados, ou seja, um conjunto de estados, por meio do qual um sistema dinâmico pode rotineiramente passar. Um sistema resiliênte vai ficar em uma determinada região do espaço de estados. Se o sistema muda para uma área completamente diferente do espaço de estados, chamamos de transformação ou mudança de regime. Uma mudança de regimes é frequentemente um processo não-linear e pode ser difícil de inverter, não sendo neste caso resiliente. Estes conceitos são explicados em detaIhes em Buschbacher (2014).

\section{REFERÊNCIAS BIBLIOGRÁFICAS}

ALMEIDA, J. et al. Territorialidade e Re-existência indígena na Fronteira Amazônica: o povo Rikbaktsa e a Terra Indígena Escondido, Mato Grosso, Brasil. Sustentabilidade em Debate, 2016.

ANDERIES, J. M.; WALKER, B. H.; KINZIG, A. P. Fifteen weddings and a funeral: case studies and resilience-based management. Ecology and Society 11(1): 21, 2006. [on-line] Disponível em: <http://www.ecologyandsociety.org/ vol11/iss1/art21/>.

ATHAYDE, S. et al. Aprendizagem colaborativa, transdisciplinaridade e gestão socioambiental na Amazônia: abordagens para a construção de conhecimento entre academia e sociedade. Revista Brasileira de Pós-Graduação, 10(21): 729-756, 2013.

BABY, A. L. T. Estudo da Dinâmica de Desmatamento do Município de Cotriguaçu - MT. Trabalho de Conclusão de Curso apresentado à Universidade do Estado de Mato Grosso, no curso de Pós-Graduação Lato Sensu em Gestão Colaborativa de Sistemas Socioecológicos Complexos na Amazônia Brasileira. 2012.

BARNES, G.; CHILD, B. Conclusions, p. 283-300. In: BARNES, G.; CHILD, B. (Ed.). Adaptive Cross-scalar Governance of Natural Resources. Routledge. 2014.

BARTELS, W. L. et al. Quem avalia resiliência e qual resiliência vale? Reflexões sobre a aplicação do Manual de Avaliação da Resiliência em uma fronteira Amazônica contestada. Sustentabilidade em Debate, 2016.

BERNASCONI, P. et al. Avaliação da Resiliência do Sistema de Médios e Grandes Proprietários Rurais de Cotriguaçu (MT, Brasil). Sustentabilidade em Debate, 2016.

BRITO, B.; BARRETO, P. A regularização fundiária avançou na Amazônia? Os dois anos do Programa Terra Legal. Belém-PA: Imazon. 2011.

BROWDER, J. O. et al. Revisiting theories of frontier expansion in the Brazilian Amazon: a survey of the colonist farming population in Rondonia's post-frontier, 1992-2002. World Development, 36(8), 1469-1492, 2008.

CARPENTER, S. R. et al. From metaphor to measurement: resilience of what to what? Ecosystems 4: 765-781, 2001.

DAVIDSON, E. A. et al. The Amazon basin in transition. Nature 481, n. 7381: 321-328, 2012.

FEARNSIDE, P. M.; LAURANCE, W. F. Infraestrutura na Amazônia: as lições dos planos plurianuais. Caderno CRH 25, n. 64: 87-98, 2012.

FOLKE, C. Resilience: The emergence of a perspective for social-ecological systems analyses. Global Environmental Change 16(3): 253-267, 2006.

GOFFMAN, E. Frame analysis: An essay on the organization of experience. Harvard University Press. 1974.

HOLLING, C. S. Resilience and stability of ecological systems. Annual Review of Ecology and Systematics 4: 1-23, 1973.

HOLLING, C. S.; GUNDERSON, L. H. Resilience and adaptive cycles, p. 25-62. In: GUNDERSON, L. H.; HOLLING, C. S. (Ed.). Panarchy: understanding transformations in human and natural systems, Washington: Island Press, 2002. 
HOLLING, C. S.; MEFFE, G. K. Command and control and the pathology of natural resource management. Conservation Biology, 10: 328-337, 1996.

ICV. Mato Grosso apresenta programa-piloto de REDD em Copenhague, 2009.

Disponível em: <http://www.icv.org.br/site/2009/12/15/mato-grosso-apresenta-programa-piloto-de-redd-emcopenhague/>. Acesso em: 10 mar. 2015

LENTINI, M. W. (Coord.). Diagnóstico do Setor Florestal de Cotriguacu, Estado de Mato Grosso. Relatório Final. 2010. Disponível em: <http://www.icv.org.br/site/wp-content/uploads/2013/08/10437relatorio_florestal_cotriguacu_ift_icv.pdf>. Acesso em: 10 mar. 2015.

LEVIN, S. A. Self-organization and the emergence of complexity in ecological systems. Bioscience 55, n. 12: 10751079, 2005.

LITTLE, P. Amazonia. Territorial Struggles on Perennial Frontiers. The Johns Hopkins University Press, Baltimore and London. 2001.

LIU, J. et al. Complexity of coupled human and natural systems. Science, 317(5844): 1513-1516, 2007.

MACEDO, M. N. et al. Decoupling of deforestation and soy production in the southern Amazon during the late 2000s. Proceedings of the National Academy of Sciences 109, n. 4: 1341-1346, 2012.

MAIA, H. et al. Avaliação do Plano de Prevenção e Controle do Desmatamento na Amazônia Legal: PPCDAm: 20072010. 2011. Disponível em: <http://repositorio.ipea.gov.br/bitstream/11058/884/1/IPEA_GIZ_Cepal_2011_Avaliacao\%20PPCDAm\%202007-2011_web.pdf>. Acesso em: 10 mar. 2015

NEPSTAD, D. et al. The End of Deforestation in the Brazilian Amazon.

Science, v. 326, n. 5958 p. 1350-1351, 2009.

NEVES, E. M. S. C. Política ambiental, municípios e cooperação intergovernamental no Brasil. Estudos Avançados, 26.74: 137-150, 2012.

OJEDA, I. Reforma agrária perde fôlego na agenda nacional. Desafios de Desenvolvimento, 75. IPEA. 2012. Disponível em: http://www.ipea.gov.br/desafios/index.php?option=com_content\&view=article\&id=2866:cati$\mathrm{d}=28 \&$ Itemid=23>. Acesso em: 10 mar. 2015.

OLIVAL, A. 2016. A resiliência em assentamentos rurais: uma experiência na região norte de Mato Grosso. Sustentabilidade em Debate, 2016.

PETERSON, G.; CUMMING, G.; CARPENTER, S. Scenario planning: a tool for conservation in an uncertain world. Conservation Biology, 17 p. 358-366, 2003.

REDMAN, C. L.; GROVE, J. M.; KUBY, L. H. Integrating social science into the long-term ecological research (LTER) network: social dimensions of ecological change and ecological dimensions of social change. Ecosystems, $7(2)$ : 161-171, 2004

RESILIENCE ALLIANCE. Assessing Resilience in Social-Ecological Systems: A Workbook for Practitioners, Version 1.1, 2007a.

Assessing resilience in social-ecological systems: Workbook for practitioners. Version 2.0. 2010. [On-line] Disponível em: <http://www.resalliance.org/files/ResilienceAssessmentV2_2.pdf>. Acesso em: 19 jul. 2016

Assessing Resilience in Social-Ecological Systems: A Workbook for Scientists, Version 1.1 2007b. [On-line] Disponível em: <http://library.uniteddiversity.coop/Transition_Relocalisation_Resilience/resilience_workbook_ for_scientists.pdf>. Acesso em: 19 jul. 2016

SCHMINK, M.; WOOD, C. H. Contested Frontiers in Amazonia. Columbia University Press, New York, 1992.

SIMÃO, B.; ATHAYDE, S. Resiliência socioecológica em comunidades deslocadas por hidrelétricas na Amazônia: o caso de Nova Mutum Paraná, Rondônia. Sustentabilidade em Debate, 2016. 
SIMMONS, C. et al. Doing it for themselves: direct action land reform in the Brazilian Amazon. World Development 38, n. 3: 429-444, 2010.

SOBREIRO, T. Dinâmica Socioecológica e Resiliência da Pesca Ornamental em Barcelos, Rio Negro, Amazonas, Brasil. Sustentabilidade em Debate, 2016.

STRICKLAND-MUNRO, J. K.; ALLISON, H. E.; MOORE, S. A. Using resilience concepts to investigate the impacts of protected area tourism on communities. Annals of Tourism Research, 37(2), p.499-519, 2010.

TURNER, F. J. The Significance of the Frontier in American History. Report of the American Historical Association for 1893, 199-227, 1893.

WALKER, B.; SALT, D. Resilience thinking: sustaining ecosystems and people in a changing world. Island Press, 2012.

WALKER, B. H. et al. Resilience, adaptability, and transformability in the Goulburn-Broken Catchment, Australia. Ecology and Society, 14(1), p.12, 2009.

VAN DER HEIJDEN, K. Scenarios: the art of strategic conversation. John Wiley \& Sons, 2005.

VERISSIMO, A. et al. Áreas Protegidas na Amazônia Brasileira: avanços e desafios. Belém/São Paulo: Imazon e ISA, 2011. 